REVISTA DE DERECHO UNED, NÚM. 12, 2013

\title{
EL ACCESO A LAS FUENTES DE INFORMACIÓN JUDICIAL EN ISRAEL Y LOS LÍMITES IMPUESTOS POR LA «SEGURIDAD NACIONAL»
}

\section{ACCESS TO THE SOURCES OF JUDICIAL INFORMATION IN ISRAEL AND THE LIMITS IMPOSED BY THE «NATIONAL SECURITY»}

\author{
Antonio Francisco Velasco SanZ \\ Profesor de Derecho \\ Centro Universitario Villanueva
}

Sumario: 1. Introducción. 2. Israel y el derecho anglosajón. 2.1 Similitudes: Selección y Attorney General. 2.2 Diferencias. 3. La censura militar. 4. El acceso a los procesos judiciales. 5. El caso de «el prisionero $\mathrm{x}$ » 6. Un precedente clave: 1953. «La voz del pueblo».6.1 Contenido del artículo. 6.2 Relevancia de la sentencia. 7. La nueva ley de difamación. 8. El lugar de Israel en el mundo en materia de libertad de expresión. 9. Conclusiones.

Resumen: El ordenamiento jurídico israelí garantiza el derecho a la libertad de expresión y de información, pero con unos límites que, en algunos casos, pueden considerarse de excesivos. Esto sucede sobre todo en procesos judiciales relacionados con la seguridad del Estado, donde incluso rige la «censura militar». En el presenta trabajo se analiza este problema, así como la labor de los periodistas en relación con los procesos judiciales. A pesar de que ha habido cierta evolución, los límites a la libertad de prensa son muchos, aunque en el entorno geográfico del Estado de Israel, se trata del país que más libertad permite en este ámbito.

Abstract: The israeli legal system guarantees the freedom of expression and information, but with limits that, in some cases, may 
be considered excessive. This happens especially in judicial proceedings related to the security of the State, where even governs the «military censorship». The present paper analyzes this problem, as well as the work of journalists regarding the judicial proceedings. While there has been some evolution, the limits to the freedom of the press are many, but in the geographical environment of the State of Israel, it is the country that allows more freedom in this area.

Palabras clave: Libertad de información, censura, información judicial.

Keywords: Freedom of information, censorship, judicial information.

Recepción original: 18/06/2013

Aceptación original: 19/06/2013

\section{INTRODUCCIÓN}

Como principio general, el Estado de Israel, que no cuenta con una Constitución escrita, garantiza en sus leyes el derecho a la libertad de expresión y de información, pero también existen unos límites en determinadas áreas que, a priori, pueden parecen más que excesivos, sobre todo en lo que afecta a procesos judiciales relacionados con la seguridad del Estado, donde incluso a día de hoy rige la «censura militar». A ésta deben acudir los medios de comunicación cuando «duden» respecto a si una determinada información puede o no afectar a esa parcela, con el fin de poder evitar posteriormente posibles sanciones. La existencia de límites a la libertad de expresión y/o información, en todo caso, no es algo excepcional.

Como sabemos, en muchos países, las propias legislaciones o la jurisprudencia -el sistema de precedentes en el caso inglés- establecen esas medidas, e incluso el propio Tribunal Europeo de Derechos Humanos lo considera adecuado al Convenio, siempre que esas restricciones sean justas, proporcionales y justificadas. Tal como establece su artículo 10.2 del Convenio, «el ejercicio de estas libertades, que entrañen deberes y responsabilidades, podrá ser sometido a ciertas formalidades, condiciones, restricciones o sanciones previstas por la ley, que constituyan medidas necesarias, en una sociedad democrática, para la seguridad nacional, la integridad territorial o la seguridad pública, la defensa del orden y la prevención del delito, la protección de la salud o de la moral, la protección de la reputación o de los derechos ajenos, para impedir la divulgación de informaciones». 
Por tanto, no hay nada que objetar a que Israel tenga en su normativa restricciones a ese derecho fundamental. Lo esencial será, al igual que en los demás casos, analizar si las mismas son proporcionales, justas y están justificadas. Y aquí es donde surgen serias dudas. Antes de adentrarnos en algunos aspectos concretos relacionados con nuestro ámbito de estudio, nos parece necesario conocer el organigrama del Poder Judicial en Israel, en el que, adelantémoslo ya, se nota claramente la influencia del sistema anglosajón. Como primera nota característica, podemos observar que existen tribunales «civiles» y tribunales «religiosos» y dentro de estos los «judíos» y los «no judíos». Estos tribunales religiosos se van a encargar sobre todo de dirimir cuestiones o conflictos matrimoniales. Esta división es algo que no ocurre en el resto de sistemas analizados. La razón no es otra que el Estado de Israel se rige, entre otras, por la ley religiosa. No se puede olvidar que hablamos de un estado confesional, donde el judaísmo desempeña un papel principal en todas las áreas de la sociedad israelí.

De esta forma, el Derecho de este Estado va a configurarse como una mezcla, por un lado, del Derecho anglosajón, del sistema del Common Law, por la influencia de ese Derecho que fue el imperante desde 1917 a 1948, año en que Israel logra su independencia; por otro lado, del Derecho francés, por el dominio del imperio Otomano de Palestina durante más de 400 años; y, en tercer lugar, del Derecho Hebreo, ya que todo lo relativo a los matrimonios y divorcios entre judíos se va a regir por la jurisprudencia del Tribunal Rabínico, es decir de un tribunal religioso. Esta mezcla es lo que va a hacer «peculiar» el sistema jurídico de este Estado.

Sobre esta cuestión, el ex profesor y ex Presidente de la Corte Suprema de Justicia Sharon Barak, sostiene que «(...) El Sistema Jurídico Israelí no pertenece a la Cultura del Derecho Hebreo, sino que esta cultura influye sobre su configuración. Aparentemente, nuestro sistema jurídico pertenece a la cultura jurídica occidental ${ }^{1}$ y no obstante la semejanza con otros sistemas Jurídicos (...) constituimos un sistema con estilo propio».

En la cúspide del sistema judicial israelí se encuentra la Corte Suprema, que es la encargada de resolver las apelaciones sobre resoluciones a los recursos contra decisiones de los tribunales de apelación, y, junto a ello, tiene legitimidad para fallar sobre la legalidad o

1 BARAK, AHARON BARAK: El Sistema Jurídico en Israel: su tradición y su cultura, (Traducción al Español realizada por el Colegio de Abogados de Israel), 2005, págs. 7 y 15 . 
no de las decisiones que adopte el Gobierno. Está integrada por doce jueces, con un presidente a la cabeza, y tienen la potestad de ordenar reabrir un procedimiento penal incluso cuando se trate de sentencia firme, aunque, en todo caso, ello no puede conllevar una pena más agravada al condenado. El sistema de tribunales, por su parte, actúa a tres niveles: la citada Corte Suprema de Justicia, los Tribunales de Distritos y los Tribunales de Magistrado.

En concreto, la Corte Suprema ejerce tres funciones principales: Tribunal Supremo, Tribunal de Apelación, pero también de una especie de "Tribunal Constitucional», ya que será el encargado de dirimir si una determinada norma se ajusta a las leyes básicas del Estado de Israel, que son las que conforman el núcleo del ordenamiento de las distintas áreas que regulan los tres poderes.

El Gobierno de Israel sostiene sin duda alguna que el Poder Judicial que rige goza de una absoluta independencia, lo que garantiza que sus integrantes no van a estar sometidos a ningún tipo de control, y, consecuencia de ello, es la defensa de los derechos civiles de los ciudadanos. Esta independencia viene recogida en la «Ley Básica: El Sistema Judicial» donde se afirma lo siguiente: "[una] persona a quien se le otorga poder judicial no debe, en cuestiones judiciales, estar sujeta a ninguna potestad fuera de la ley ${ }^{2}$. Además de su independencia sustancial, los jueces "gozan de una amplia independencia personal, que comienza con el procedimiento para su selección y continúa durante todo su período en el cargo: los jueces son seleccionados por la Comisión de Selección Judicial» ${ }^{3}$, que está compuesta por nueve miembros: el Ministro de Justicia (presidente), otro ministro del gabinete, el Presidente de la Corte Suprema, otros dos jueces de la Corte Suprema, dos miembros de la Knéset (Parlamento) y dos representantes del Colegio de Abogados de Israel. La mayoría de los miembros de la comisión son abogados de profesión: los tres jueces de la Corte Suprema y los dos representantes de la profesión legal. Un candidato puede ser propuesto por el Presidente de la comisión, el Presidente de la Corte Suprema o tres miembros cualesquiera de la comisión. Se requiere el voto mayoritario de los miembros de la comisión para el nombramiento de un candidato.

2 Artículo 1 de la «Ley Básica: El Sistema Judicial» de Israel (Basic Law: The Judiciary, disponible en http://www.knesset.gov.il/laws/special/eng/basic8_eng.htm ).

3 Fuente: Ministerio de Asuntos Exteriores de Israel. Puede encontrarse información básica sobre el sistema judicial en Español en la página del Ministerio mencionado: http://www.mfa.gov.il $/ \mathrm{mfa} / \mathrm{mfaes} /$ facts $\% 20$ about $\% 20 \mathrm{israel} /$ pages $/ \mathrm{el} \% 20$ sistema $\% 20$ judicial.aspx 
Una vez que el candidato ha obtenido el refrendo de la comisión y es nombrado juez, su designación es permanente y sólo expira con el «retiro» obligatorio» a los 70 años. Al igual que los parlamentarios, los jueces gozan de una inmunidad similar y solamente pueden ser removidos por una decisión de la Corte Disciplinaria, integrada por jueces nombrados por el presidente de la Corte Suprema, o por decisión de la Comisión de Selección Judicial, a propuesta del Ministro de Justicia, o del Presidente de la Corte Suprema. La decisión de la comisión debe estar apoyada por siete de sus nueve miembros.

La composición del Poder Judicial en Israel viene configurada en la Ley del Sistema Judicial de Israel, donde se recoge no sólo este aspecto, sino también quiénes pueden acceder al mismo -sólo ciudadanos del Estado- la edad de jubilación, sistema de retribuciones, etc.

En cuanto a la estructura del Poder Judicial, éste reside en los tribunales siguientes:

- El Tribunal Supremo.

- Tribunales de distritos. En Israel existen 5 tribunales de distrito: Beer-Sheva, Haifa, Jerusalén, Nazaret y Tel-Aviv. Sus sentencias son susceptibles de apelación ante la Corte o Tribunal Supremo.

- Juzgados de Paz o tribunales de magistrados. Constituyen la primera instancia en los ámbitos Civil y Penal. Sus resoluciones son recurribles ante el tribunal de distrito correspondiente

- Otros tribunales designados por la ley. Entre éstos se encuentran, por ejemplo, del Tribunales de Familia o los de Menores, cuyas vistas (al igual que sucede en el resto de países analizados) se celebran siempre a puerta cerrada.

Además, le mencionada ley recoge que el Poder Judicial reside también en los siguientes:

- Tribunales religiosos religioso (beit din). Se encargan de dirimir todo lo relacionado al matrimonio, divorcio, etc., entre cónyuges de religión judía, aspectos para los cuales tienen «exclusividad» y sin que puedan intervenir los tribunales «civiles».

- Cualquier otro tribunal (beit din) previsto por la ley. 


\section{ISRAEL Y EL DERECHO ANGLOSAJÓN}

\subsection{Similitudes: selección y Attorney General}

Ya habíamos comentado anteriormente que el Derecho israelí tenía influencias del anglosajón, del sistema de Common Law. Ello se observa en dos aspectos esenciales: la inexistencia de una Carrera Judicial propiamente dicha y la presencia de una figura similar a la que existe en Reino Unido, como es la existencia del Attorney General.

En cuanto la Carrera Judicial, al igual que en Reino Unido y los países de su influencia, no existe como tal, es decir, no hay ninguna «escuela judicial» a la que deban acudir los que previamente hayan pasado unas determinadas pruebas. Por el contrario, se seleccionan entre aquellos candidatos profesionales que pertenezcan a cualquiera de las profesiones jurídicas (abogados, procuradores, profesores especializados en áreas del Derecho...). Será la comisión formada al respecto la que designe a quienes deben integrar los distintos escalafones del Poder Judicial.

Junto a ello, encontramos la presencia del Attorney General, que es el asesor legal del Gobierno, una de las funciones que también desempeña en Reino Unido ${ }^{4}$ (aunque existe una diferencia a la que aludiremos en el punto siguiente). Esta figura, quien la ocupe, se encuentra a la cabeza de la pirámide del sistema legal del Poder Ejecutivo, pero, a la vez, es el encargado de defender que las leyes que se aprueban no vulneren las leyes que rigen el Estado.

No obstante, sobre su independencia respecto al Poder Ejecutivo surge una duda, y no es otra que la siguiente: su designación la realiza directamente el primer ministro del Estado; y ello puede de alguna forma, aunque teóricamente no sea así, influir a la hora de adoptar algunas decisiones que afecten directamente al Gobierno. Además, a ello hay que añadir que forma parte del Consejo de Ministros, con lo que su independencia puede quedar aún más marcada por la sombra de esa duda.

Sin embargo, no depende ni del primer ministro ni del titular de la Cartera de Justicia, y, en principio, goza de absoluta independencia, aunque no podemos dejar de señalar las dudas expresadas anteriormente. La relevancia que adquiere esta figura es tal que es

4 Sobre el Attorney General en el Reino Unido, pueden verse más detalles en: https://www.gov.uk/government/ministers/attorney-general 
considerada «uno de los pilares de la democracia israelí y su independencia es crucial para nuestra democracia ${ }^{5}$.

\subsection{Diferencias}

En relación con los aspectos anteriormente mencionados, y que son comunes al Derecho Anglosajón, hay unas diferencias a las que aludimos a continuación. En primer lugar, en el Derecho israelí no hay procesos por Jurado, mientras que en Reino Unido prácticamente todos son decididos por jurados populares. En Israel, por el contrario, el juez es el único con poder de decisión en un proceso judicial. No existe, por tanto, participación ciudadana a la hora de dirimir una causa.

En cuanto a la figura del Attorney General, encontramos dos diferencias sustanciales: En Israel, además de ser el asesor legal del Gobierno, es igualmente el principal responsable de la Fiscalía, y es quien decide sobre la postura final del Ministerio Público en los procesos. En Reino Unido, por el contrario, existe un fiscal general independiente del Attorney General. Esta separación también se ha tratado de llevar a cabo en Israel, aunque, por el momento, no se ha consolidado.

Sobre la posibilidad de iniciar un proceso contra los medios de comunicación y de instar directamente a que no se publiquen determinadas informaciones, ya que de lo contrario dará lugar a un proceso por vulnerar la ley de desacato, como sucede en Reino Unido, tal opción no existe en Israel, donde el Attorney General sí tiene posibilidad de acudir a un juez para que éste acuerde por una resolución que no se puede publicar determinada información, lo que se conoce como una «orden de prohibición de publicidad».

\section{LA CENSURA MILITAR}

En Israel, quizás por las circunstancias políticas en las que vive, tiene una gran importancia e influencia el ejército, uno de los pilares fundamentales, quizás el primero de ellos, sobre los que se asienta la seguridad nacional. Por ello, tiene una especial relevancia en todos los sectores de la sociedad, y, por tanto, también en lo que afecta a los medios de comunicación.

5 Haiat, Lior, consejero y portavoz de la Embajada de Israel en España. Entrevista personal mantenida en la Embajada de Israel el 26 de abril de 2013. 
A este respecto, el Ejército, si tiene conocimiento de que determinada información ha llegado a poder de los medios de comunicación y ésta puede incidir negativamente en lo que se engloba como «seguridad nacional», puede acudir al juez para que frene esa difusión. En principio, los medios pueden publicar todo aquello que consideren relevante para la sociedad, pero si hay algunos aspectos que pueden ser «cuestionables» lo mandan a la «censura militar» para que ésta decida si ello afecta o no a la seguridad nacional. Si la «censura» entiende que no debe publicarse, el medio de comunicación puede acudir a un juez para que resuelva. "Es responsabilidad del medio de comunicación acudir o no a esa censura» ${ }^{6}$.

Pero el medio puede publicar directamente la información controvertida sin solicitar la opinión de la «censura militar». En estos casos, el medio se arriesga a ser demandado por daños a la seguridad del Estado, lo que podría suceder, por ejemplo, con la difusión de la identidad de un agente de los servicios secretos israelíes.

\section{EL ACCESO A LOS PROCESOS JUDICIALES}

Como norma general, los periodistas especializados en información judicial pueden acudir a presenciar libremente los procesos que sean públicos, pues, como hemos indicado, hay algunos que, por circunstancias excepcionales, se celebran a puerta cerrada. Estas limitaciones a la publicidad de los procesos vienen recogidas en la propia ley judicial, y entre otras, figuran las causas que se refieran a la Seguridad Nacional, protección de los menores y Víctimas de delitos sexuales.

Para poder acceder a una sala de vistas, es necesario que el profesional de la comunicación cuente con una acreditación concedida por la Corte israelí, un permiso otorgado por la Administración de los Tribunales Esto se refiere sobre todo a los miembros de la prensa que cubren los asuntos judiciales y los distintos tribunales repartidos en cada uno de los diferentes condados ${ }^{7}$.

Sobre esta cuestión, Re'em Segev, profesor titular en la Facultad de Derecho de la Universidad Hebrea de Jerusalén, y uno de los mayores expertos en esta materia en Israel, realiza un somero estudio

6 HAIAT, Lior, consejero y portavoz de la embajada de Israel.

7 Respuesta recibida a una consulta por correo electrónico del Ministerio de Justicia de Israel, el 7 de febrero de 2013. 
en uno de sus libros ${ }^{8}$, en los que viene a analizar los conflictos entre la libertad de expresión y el acceso a los procedimientos judiciales, y las justificaciones para que se puedan adoptar determinadas «injerencias» en el primero de esos derechos, y que se resumen en evitar cualquier "perturbación» que pueda indicar de forma más o menos directa en el devenir del procedimiento, de su resolución.

En opinión del mencionado profesor, como regla general, sería necesario «limitar» el derecho a la libertad de expresión sólo cuando de las informaciones se pueda poner en peligro de forma «significativa» la «corrección del procedimiento judicial», supuestos en los que sí estaría justificada la imposición de límites como medidas cautelares y sólo para casos especialmente graves.

\section{EL CASO DE «EL PRISIONERO X»}

Vamos a analizar ahora un caso reciente, donde podremos comprobar el silencio que rodea a aquellos procesos en los que se consideran que pueden afectar a la seguridad del Estado. Es lo que sucedió con el «prisionero X».

En el mes de febrero de 2013, salió a relucir en los medios de comunicación de prácticamente todo el mundo, lo ocurrido con el conocido como "prisionero $\mathrm{X}$ ». Se trataba de un condenado que había aparecido ahorcado en su celda en 2011. Las informaciones contribuyeron a conocer la historia de ese recluso. Se trataba de un ciudadano con doble nacionalidad israelí y australiana que había sido juzgado y condenado bajo la más absoluta prohibición de difusión de cualquier aspecto relacionado con ese proceso, al entender que afectaba al principio de seguridad nacional, pues se trataba de un miembro de los servicios secretos de Israel. Durante ese juicio, el procesado, del que no se llegó a facilitar su identidad, contó con tres abogados defensores.

En este caso, el Estado, a través de su representación legal, acudió al juez y solicitó que dictase una orden de prohibición respecto a cualquier publicidad relacionada con los hechos investigados. El juez accedió a esa solicitud y fue lo que determinó que durante años no se diese ningún dato al respecto en los medios de información del país, aunque, paradójicamente, en Australia sí se habían difundido informaciones al respecto.

8 Re'em Segev: Sub Judice: Freedom of Expression in Matters under Adjudication, Democracy Institute, 2001. Jerusalén. 
La primera resolución al respecto se produjo el 16 de diciembre de 2010, dictada por el tribunal de paz de la ciudad de Reshom Letzion, donde se acordó la imposibilidad de ningún tipo de publicidad sobre esta causa. Un periodista y dos canales de TV apelaron ese fallo, pero la decisión fue confirmada. El argumento esgrimido por el Gobierno, y refrendado por el tribunal, se reducía esencialmente a que con esa medida se garantiza la privacidad del fallecido y de su familia, así como datos que podían afectar a la seguridad nacional. De esta forma, daba prevalencia a estos aspectos sobre el interés público que existía por conocer lo ocurrido realmente con este preso.
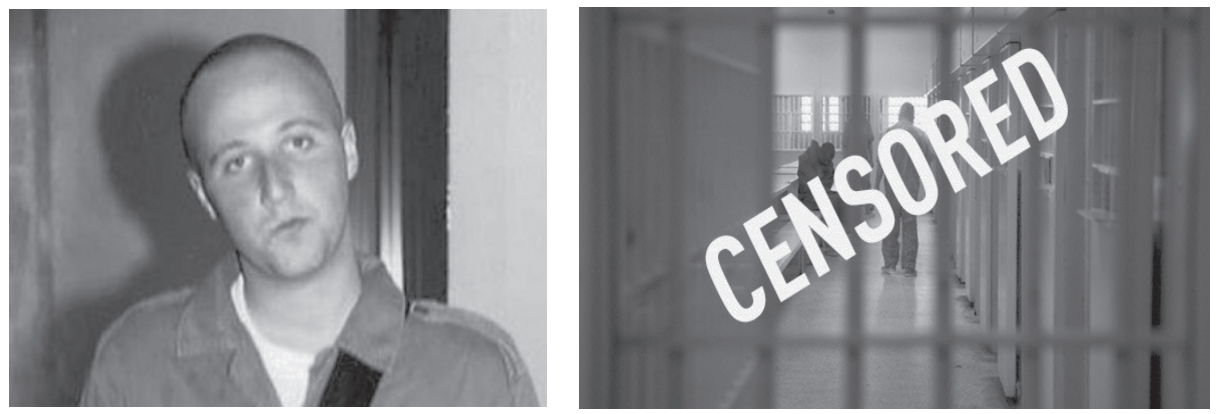

Esta era la situación que existía -prohibición de cualquier tipo de publicación al respecto- cuando el caso salta a los medios de comunicación internacionales. Así, el pasado 19 de febrero de 2013, se produce una resolución judicial que va a propiciar un giro relevante en lo que se refiere al derecho a la información en este proceso ${ }^{9}$, cuando una magistrada revocó la decisión anterior y ordenó al Gobierno que facilitase la información respecto a determinados puntos del proceso.

En esta decisión vemos dos aspectos relevantes. En primer lugar, que una jueza ordena al Gobierno que tenga que facilitar a los medios de comunicación determinada información que había sido vetada anteriormente; $y$, en segundo lugar, se incide en que no toda información es publicable, ya que hubo no pocos puntos de las decisiones previas que continuaron bajo secreto. ¿Por qué una parte de la información sí se puede publicar y otra no? ¿Qué se busca al impedir mantener bajo prohibición que los ciudadanos conozcan determinados aspectos de ese proceso?

La decisión de esta magistrada se produjo después de que llevara a cabo una investigación sobre la muerte del citado prisionero y llegase a la conclusión de que no existió ningún tipo de negligencia y que se

9 Auto de la juez Dafna Belteman de 19 de febrero de 2013. 
trató de un suicido. Esta fue la obligación que estableció la magistrada que debía publicarse, en contra de lo que había sostenido el Gobierno, quien pidió que se mantuviesen todas las reservas al respecto.

\section{UN PRECEDENTE CLAVE: 1953. «LA VOZ DEL PUEBLO»}

Para entender mejor la situación de la información judicial en Israel, sus límites y causas del mismo, hay que remontarse a una resolución del Tribunal Supremo dictada en $1953^{10}$, apenas seis años después de que este Estado alcanzara su independencia; eran años en los que se aprobaban las leyes básicas que van a regular el funcionamiento de la sociedad hebrea.

En esa resolución, cuya doctrina es la que permanece vigente todavía, y, por tanto, marca la línea jurisprudencia al respecto, el Tribunal Supremo Israelí va a analizar dos cuestiones fundamentales: la libertad de prensa, por un lado, y todo lo relativo a informaciones que pueden afectar la seguridad nacional, por otro. Se trataba de la resolución «Kol Haam vs Ministro del Interior» y el origen de toda la cuestión se centraba en la publicación en citado periódico «Kol Haam» («La Voz del Pueblo»), órgano oficial del Partido Comunista, de un artículo titular «Haaretz», que previamente se había difundido en el diario «Haaretz». Se trata de la sentencia que «para algunos marcó el advenimiento del activismo judicial del Tribunal Supremo Israelí» ${ }^{11}$.

\subsection{Contenido del artículo}

El artículo, que fue publicado tanto en su versión hebrea como árabe, hacía referencia a que en una hipotética guerra entre Estados Unidos y Rusia, por el conflicto de Corea, Israel apoyaría a los americanos con el envío de unos 200.000 soldados. Esta información no estaba lo más mínimamente contrastada. El Gobierno entendió que con esa información lo que se buscaba realmente era un conflicto del Ejecutivo con los ciudadanos.

Por ello, el ministro del Interior, invocando una ley de 1933 que aún permanece efectiva en territorio israelí, creada en la época del mandato británico, que le otorga poderes para cerrar un periódico si «supone un peligro para el orden público, decidió clausurar el mencionado periódico durante 10 días y con la advertencia de que sería

10 Sentencia del Tribunal Supremo de Israel de 16 de marzo de 1953.

11 TALES PEREIRA, J.A. «De actor secundario a actor principal: El Tribunal Supremo y la "Revolución Constitucional" en Israel», en Revista de Derecho Político, n. ${ }^{\circ} 69,2007$, págs. 223-259. UNED. 
definitivo si con sus informaciones se dañaba «la paz o seguridad o de la sociedad israelí».

El mencionado medio de comunicación apeló al Tribunal Supremo, en su condición de «Tribunal Constitucional» y garante de que las normas y decisiones gubernamentales respeten las leyes básicas del Estado, por entender que con la resolución del ministro del Interior se había dañado gravemente la libertad de expresión.

La sentencia, de la que fue «relator» o ponente el juez Shimon Agramat, partía de la declaración de independencia de Israel, que no tiene rango de Constitución ni de ley, para concluir que la libertad de expresión es la «libertad superior» en el sistema judicial israelí, a la vez que ponderó los dos conflictos existentes: libertad de prensa y seguridad nacional. Al respecto, concluyó que para poder penalizar el primero de esos derechos era necesaria una constatación "cercana al 100\%», "casi certeza» de que la información cuestionada iba a dañar la seguridad nacional, y no de «mera probabilidad». De esta forma, resolvió que la decisión del ministro del Interior había sido desproporcionada y suponía un ataque a la libertad de expresión.

\subsection{Relevancia de la sentencia}

En esta sentencia se establecía la premisa desde la que debe partir el Gobierno a la hora de poder acordar una medida tan drástica como el cierre de un medio de comunicación, que no es otra que la necesidad de que los supuestos ataques contra la seguridad nacional derivados de la publicación de determinadas informaciones deben estar más que contrastados.

La trascendencia de esta resolución va mucho más allá del caso concreto que resolvió, pues se trata de la primera sentencia del Tribunal Supremo de Israel subsiguiente a la aprobación por el Knesset, en agosto de ese mismo año, de la llamada "Ley de los Jueces», concretando, verdaderamente, "por primera vez desde la fundación del Estado de Israel, una garantía legal efectiva de la independencia del Poder Judicial» ${ }^{12}$.

Pero, a la vez, pone de manifiesto la «facilidad» con que el Ejecutivo, a través del ministro del Interior, puede cerrar un medio de comunicación. Y este caso no ha sido una excepción, pues reciente-

12 TALES PEREIRA, J.A. «De actor secundario a actor principal: El Tribunal Supremo y la "Revolución Constitucional" en Israel», en Revista de Derecho Político, n69, 2007, UNED, págs. 223-259 
mente, se ha adoptado esa misma medida respecto a una emisora de radio y dos cadenas de televisión. Así, en noviembre de 2011, el Ministerio de Comunicación ordenó el cierre de la radio 'Kol Hashalom' -en español, 'todo por la paz'-, situada en la Jerusalén oriental ocupada, acusándola de no tener licencias e «incitar al odio hacia Israel». Apenas unos meses después, en marzo de 2012, las fuerzas de seguridad israelíes confiscaban los aparatos técnicos de dos televisiones que emitían desde territorio palestino. En este caso, el argumento era también que no disponían de licencias para emitir y que, además, perturbaban las comunicaciones aéreas.

\section{LA NUEVA LEY DE DIFAMACIÓN}

En relación con la libertad de expresión, tenemos que aludir, aunque sea brevemente, a la nueva «Ley de Difamación», aprobada por el Parlamento de Israel el 22 de noviembre de 2011, y que desde algunos sectores se considera que se trata de un nuevo paso restrictivo en esta materia. Esta ley tiene dos puntos esenciales: por un lado, se fija una indemnización mínima de 53.000 euros para quienes un medio de comunicación haya difamado, lo que debe reconocerse en sentencia, y, por otro lado, y es quizás lo más relevante, que no será necesario que se acredite que el afectado haya sufrido algún tipo de daño por esa información. Bastará con que se acredite que la información es falsa o errónea para que se pueda demandar a un medio de comunicación y obtener la indemnización correspondiente.

Una de las cuestiones más llamativas es que se produce con esa ley lo que se conoce como «la inversión de la prueba», es decir, corresponderá al medio de comunicación demandado demostrar que la información en cuestión es veraz, pues el afectado-interesado no tiene que demostrar absolutamente nada. Hasta ahora, sucedía justamente lo contrario, es decir, que quien pretendiese recibir una indemnización por una información debía demostrar que había sufrido un daño real por la misma, como consecuencia de que no se ajustaba a la realidad.

En el debate parlamentario, el primer ministro hebreo, Benjamin Netanyahu, defendió la ley y argumentó que «nadie nos va a dictar qué pensar, escribir, investigar o emitir. Pero la libertad de expresión debe ser garantizada para todos los sectores de la sociedad». En esta línea, se defendió de las críticas al asegurar que «Nadie tiene el derecho a la calumnia», dijo Netanyahu al pleno de la Knesset. «Este 
proyecto de ley tiene la intención de volver a la publicación de la verdad» ${ }^{13}$.

Pero parece que no se va a quedar en las demandas a medios de comunicación, pues muy recientemente, en concreto, el pasado 7 de mayo de 2013, el comité ministerial israelí para Legislación ha aprobado la «ley Jenin Jenin», que permitirá, de refrendarse definitivamente en el Parlamento (lo que no había sucedido a la hora de redactar este trabajo) que una persona o grupo de personas sean demandadas por calumniar al Ejército a través de una demanda civil, en el marco de la ley de difamación existente.

El proyecto de ley fue presentado por el parlamentario Yoni Sitbon, miembro del partido ultranacionalista Bayit Yehudi, quien lo justificó en que «todos los que están desplegados para proteger a los ciudadanos de Israel y sus fronteras son los que se ven constantemente vilipendiados a través de los medios de comunicación». El ministro de Justicia, Tzipi Livni, se opuso a esa proposición por entender que la misma podría «dañar» la libertad de expresión ${ }^{14}$.

Lo cierto es que la Ley de Difamación ya aprobada ha recibido no pocas críticas tanto por medios de comunicación como por asociaciones de periodistas, Reporteros sin Fronteras, etc., que ven en ella un «palo» más en la «rueda de la libertad de expresión». En mi humilde opinión, me parece que sería necesario acreditar que una información ha podido causar un daño, pues por el simple hecho de que no sea totalmente cierta no por ello es merecedora de sanción y que este tipo de leyes no contribuyen, precisamente, a fortalecer un derecho fundamental, reconocido por otro lado, en la leyes básicas de Israel y por su Tribunal Supremo, como es el de la libertad de expresión. Me parece interesante resaltar algo ya mencionado anteriormente: la existencia de límites a ese derecho es algo natural y lógico, pero, tal como ha reconocido el Tribunal Europeo de Derechos Humanos (ver capítulo relativo al mismo), se debe partir de la prevalencia del mismo y no al revés, como parece desprenderse del contenido de esta ley.

Baste citar aquí, a título de mero ejemplo, que nuestro Tribunal Constitucional o Tribunal Supremo, tiene establecida una consolidada jurisprudencia donde lo relevante es que se cumpla el requisito de veracidad en la información, lo que no implica que tenga que corresponder con la verdad absoluta. Como señala una muy reciente sentencia de la Sala Civil del Tribunal Supremo «El deber de veracidad no puede ser entendido como exigencia de verdad absoluta, pues la libertad

13 Difundida por varias agencias de prensa el 22 de noviembre de 2011.

14 Agencia Europa Press. Jerusalén, 7 de mayo de 2013. 
de información es compatible con la existencia de errores e inexactitudes (...) que no alteran el núcleo de la información, la esencia de lo informado» "15; o, como ha señalado el máximo garante de la Constitución, "la información rectamente obtenida es digna de protección, aunque su total exactitud sea controversible o se incurra en errores circunstanciales que no afectan a la esencia de lo informado» ${ }^{16}$.

\section{EL LUGAR DE ISRAEL EN EL MUNDO EN MATERIA DE LIBERTAD DE EXPRESIÓN}

Por último, unas breves líneas para «situar» a Israel en el ámbito internacional en lo que se refiere a la libertad de expresión. De los países analizados, es el que ocupa peor lugar en el «ranking» elaborado por Reporteros Sin Fronteras ${ }^{17}$ respecto a los «indicadores» de la libertad de prensa, pero, sin embargo, está muy por encima de los países de su alrededor.

Sobre este punto, el informe de RSF reconoce que los periodistas de Israel «disfrutan de libertad de expresión, a pesar de la existencia de la censura, pero el país ha caído varios puestos por sus ofensivas contra los profesionales de la información en los territorios palestinos». En concreto, mientras que en el año 2011 acabó en el puesto 92, el pasado año retrocedió hasta el 112.

Esta asociación justifica este descenso, entre otras medidas, en la existencia de la «detención administrativa» para «tener detenidos a centenares de ex-activistas y periodistas palestinos».

Sin embargo, este punto no puede analizarse de forma aislada a la situación que vive Israel, en la que, como hemos reiterado, la seguridad nacional es la premisa fundamental a la hora de adoptar determinadas medidas que pueden suponer una restricción en derechos fundamentales, como puede ser la libertad de expresión.

El problema, como sucede con todos los países, radica en la proporcionalidad, y este es el aspecto que ha criticado recientemente el relator especial de Naciones Unidas sobre la Libertad de Expresión y Opinión, Frank La Rue, tras la reciente aprobación de la ya expuesta Ley de Difamación, el entender que suponer limitar por ley la libertad de expresión, además de aumentar las penas por esos delitos. Tras visitar Israel y los territorios ocupados el pasado mes de junio

15 STS 163/2013, de 20 de marzo.

16 SSTC 21/2000, de 31 de enero, y 126/2003, de 30 de junio.

17 www.rsf-es.org, Informe anual presentado en Madrid el 30 de enero de 2013. 
de 2013, La Rue expresó «su preocupación por los intentos de Israel de disminuir el espacio para la crítica en el propio país en relación a sus políticas y prácticas de ocupación, incluyendo la adopción de una serie de leyes muy restrictivas en el Knesset» ${ }^{18}$. A la vez, recomienda al gobierno israelí que acabe con la figura del Censor, y que las restricciones a la libertad de expresión justificadas en el supuesto atentado contra la seguridad nacional sean reguladas por Ley.

\section{CONCLUSIONES}

El aspecto quizás más relevante y positivo es el reconocimiento, incluso por asociaciones como RSF, de que los profesionales de la comunicación «disfrutan de libertad de expresión», algo que no podría pronunciarse respecto a la situación por la que atraviesan los periodistas en países de su entorno más próximo. En palabras del ex político del Partido Laborista de Israel y actual profesor de la Universidad de Bar Llan Gerald M. Steinberg, «Ninguna otra democracia puede presumir de tener una mayor libertad de expresión, a pesar de más de seis décadas de guerra y terrorismo, amenazas de aniquilación....Israel protege sistemáticamente los derechos de libertad de expresión y de protesta de sus minorías...una prensa libre y muy crítica» a las políticas del Gobierno, las cuales «no serían posibles en un país sin libertad de expresión» ${ }^{19}$.

No es éste el lugar para discutir o refrendar tal manifestación, pero en ella vemos nuevamente cómo aparecen términos como «terrorismo», «amenazas» o "aniquilación», es decir conceptos vinculados a la seguridad nacional de Israel. Al margen de ello, hay un dato objetivo: es el país que tiene mejor valoración en materia de libertad de expresión si se compara con, por ejemplo, Jordania, Egipto, Irán, Líbano y Siria. Con estos dos últimos, mantiene enfrentamientos con frecuencia. Así, de los 177 estados analizados por Reporteros Sin fronteras en el informe al que ya aludimos anteriormente, Jordania aparece en el puesto 134 (22 puestos por debajo de Israel); Egipto, en el 158 (frente al 112 del analizado en este apartado); Irán, en el 174 (sólo en tres países los periodistas sufren más restricciones a la hora de desempeñar sus funciones); Líbano, en 131 (inferior en nueve puestos frente a Israel), y Siria, en el 176, convirtiéndose así los periodistas críticos con el Gobierno, con sus actuaciones, en auténticos «objetivos» de las autoridades.

\footnotetext{
18 Información difundida por varias agencias de prensa.

19 STEINBERG, Gerald M. «La política de las ONG, los Derechos Humanos y el Conflicto Árabe-Israelí». Israel Studies 16.2 (verano de 2011).
} 
Pero tampoco hay que pecar de un optimismo desmesurado, ni mucho menos. En primer lugar, porque, tal como hemos visto, el ministro del Interior tiene potestad para clausurar temporalmente un medio de comunicación; en segundo lugar, porque el Gobierno puede instar directamente a que no se facilite información sobre un determinado asuntos judicial; en tercer lugar, porque la censura militar supone de facto un freno a determinadas investigaciones(parece que tiene difícil justificación hoy día en un Estado democrático que pueda existir tal «control» previo a las informaciones); en cuarto lugar, porque todos las causas judiciales en las que se considere que, de alguna forma, lo que se ventile en esa sala de vistas puede, aunque sólo sea «rozar», la seguridad nacional, no es que no se pueda realizar ningún tipo de grabación, es que ni siquiera se permite el acceso de los periodistas; y nadie puede negar que la inmensa mayoría de esos juicios tienen un más que notable interés público (¿No lo tendría, por ejemplo, el del «prisionero X»?). ¿Por qué se quiere ocultar a la sociedad cómo se desarrollan esos procesos judiciales? ¿No fomenta ello las críticas a la transparencia judicial? ¿Por qué hay casos en los que ni siquiera se informa, aunque sea escuetamente, del inicio de un proceso judicial y de su posterior archivo o enjuiciamiento, y decisión final?

Son demasiadas preguntas en las que las respuestas, a buen seguro, no convencerían a los periodistas; demasiadas preguntas que frenan un derecho fundamental: el de los ciudadanos a conocer lo que sucede en los procesos judiciales que sean más «conflictivos». Una mayor apertura en esta cuestión contribuiría, sin género de dudas, a una mayor transparencia y, como consecuencia, a una mayor confianza de la sociedad en sus tribunales en procesos relacionados con la «seguridad nacional», toda vez que se transmitiría que se han desarrollado respetando todos los derechos de los acusados.

La conclusión se puede resumir en una frase: Libertad de los periodistas para ejercer sus funciones, pero con un secretismo en todo lo que afecta a procesos judiciales vinculados a terrorismo, seguridad nacional, etc. La cuestión central en este punto es que el ocultismo nunca es la solución, es el problema. 
